\title{
Sex differences in the neuroanatomy of alcohol dependence: hippocampus and amygdala subregions in a sample of 966 people from the ENIGMA Addiction Working Group
}

\author{
Sally Grace (1) ', Maria Gloria Rossetti ${ }^{2,3}$, Nicholas Allen ${ }^{4,10}$, Albert Batalla ${ }^{5}$, Marcella Bellani², Paolo Brambilla $\mathbb{E}^{3,6}$, \\ Yann Chye”, Janna Cousijn ${ }^{8}$, Anna E Goudriaan ${ }^{9}$, Robert Hester $\mathbb{B}^{10}$, Kent Hutchison ${ }^{11}$, Izelle Labuschagne $\mathbb{D}^{1}$, \\ Reza Momenan ${ }^{12}$, Rocio Martin-Santos ${ }^{13}$, Peter Rendell ${ }^{1}$, Nadia Solowij $\mathbb{1}^{14}$, Rajita Sinha $\mathbb{B}^{15}$, Chiang-shan Ray Li (i) ${ }^{15}$,

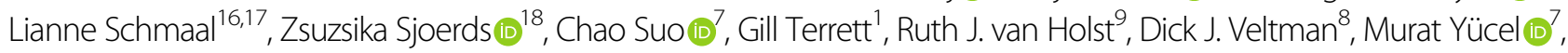 \\ Paul Thompson ${ }^{19}$, Patricia Conrod ${ }^{20}$, Scott Mackey ${ }^{21}$, Hugh Garavan ${ }^{21}$ and Valentina Lorenzetti $\mathbb{D}^{1}$
}

\begin{abstract}
Males and females with alcohol dependence have distinct mental health and cognitive problems. Animal models of addiction postulate that the underlying neurobiological mechanisms are partially distinct, but there is little evidence of sex differences in humans with alcohol dependence as most neuroimaging studies have been conducted in males. We examined hippocampal and amygdala subregions in a large sample of 966 people from the ENIGMA Addiction Working Group. This comprised 643 people with alcohol dependence (225 females), and a comparison group of 323 people without alcohol dependence (98 females). Males with alcohol dependence had smaller volumes of the total amygdala and its basolateral nucleus than male controls, that exacerbated with alcohol dose. Alcohol dependence was also associated with smaller volumes of the hippocampus and its CA1 and subiculum subfield volumes in both males and females. In summary, hippocampal and amygdalar subregions may be sensitive to both shared and distinct mechanisms in alcohol-dependent males and females.
\end{abstract}

\section{Introduction}

The burden of alcohol dependence to society is substantial, with an estimated global annual economic impact of between 210 and 665 billion US dollars arising from health consequences, workplace issues, safety, drinkdriving, and criminal costs ${ }^{1,2}$. Alcohol dependence is more common in men than in women ${ }^{3}$. However, the impact of alcohol dependence on women is particularly problematic

\footnotetext{
Correspondence: Valentina Lorenzetti (valentina.lorenzetti@gmail.com)

${ }^{1}$ Neuroscience of Addiction \& Mental Health Program, Healthy Brain and Mind Research Centre, School of Behavioural \& Health Sciences, Faculty of Health Sciences, Australian Catholic University, Melbourne, VIC, Australia

${ }^{2}$ Department of Neurosciences, Biomedicine and Movement Sciences, Section of Psychiatry, University of Verona, Verona, Italy

Full list of author information is available at the end of the article
}

as women are more likely to suffer negative consequences of drinking, and have shown an increase in recent years in their rates of alcohol dependence ${ }^{4}$. Known as the telescoping effect ${ }^{3}$ women who drink excessively on average have worse medical and mental health outcomes ${ }^{5}$, transition to dependence more quickly ${ }^{6}$, have stronger cravings ${ }^{7}$, and find it harder to quit drinking ${ }^{8}$, compared to their male counterparts. These differences may arise from distinct neurobiological mechanisms in men and women with alcohol dependence.

Current neurobiological theories of addiction emphasise brain systems underlying motivation, learning, and stress (e.g., amygdala and hippocampus), which are implicated in compulsive alcohol use, alcohol-seeking, 
and high-stress levels while craving alcohol ${ }^{9}$. However, there is a knowledge gap in the evidence informing such theories as they are largely biased by the inclusion of male-only samples, and three-quarters of published studies have failed to evaluate the interaction between alcohol dependence and $\operatorname{sex}^{10,11}$. International and national drug agencies recognise that addressing this gap is now a priority (e.g., U.S. National Institute of Health and the U.S. Federal Drug Administration) ${ }^{12}$. New evidence on sex differences within the neurobiology of alcohol dependence is required to advance existing neuroscientific theories of addiction, clarify the role of sex in the mechanisms of substance use disorders, contribute to the design of gender-tailored treatments, and inform personalised medicine.

Animal models of alcohol dependence highlight a key role of select subregions within the amygdala and hippocampus in stress, learning, and memory processes crucial for developing and maintaining addiction ${ }^{13}$. Within the amygdala, dendritic spine remodelling and glutamate function of the central nucleus are implicated in the drive for using alcohol ${ }^{9}$ and anxiety during withdrawal ${ }^{14}$ in alcohol-dependent rats. The firing of neurons within the basolateral amygdala is also involved in reward learning and the conditioned-craving for alcohol ${ }^{9,15}$, and alcoholrelated negative affect during withdrawal ${ }^{13,16}$. Additionally, sex differences in the integrity of select amygdala nuclei in alcohol administration studies are emerging. For example, basolateral and central amygdala neurons of female compared to male rats show enhanced neuronal responses to stress during ethanol self-administration, and may therefore be implicated in stress-induced alcohol seeking ${ }^{14}$. Further, glutamate neurons within the central amygdala in male compared to female animals show greater sensitivity to ethanol, and this may contribute to the higher propensity toward alcohol use observed in males ${ }^{14}$.

In relation to the hippocampus, alterations in specific subfields are implicated in alcohol dependence, intoxication, and withdrawal ${ }^{17-21}$. For example, chronic exposure to alcohol leads to the loss of pyramidal and granule cells of the dentate gyrus, CA1, and $\mathrm{CA}^{22,23}$, and to reduced neurogenesis within the dentate gyrus ${ }^{22}$. Early findings in alcohol-dependent animals also show sex differences in hippocampal subfields. Alcohol dependence and increased drug-seeking are linked to ultrastructural damage and aberrant neural activity of select hippocampus subfields in female but not male rats (e.g., dentate gyrus, CA1, and $\mathrm{CA} 3)^{22-24}$. These structural damages are associated with a range of adverse outcomes characteristic of alcohol dependence: including spatial learning and memory deficits $^{17,25-27}$, increased alcohol taking, and relapse ${ }^{20}$.

The above-mentioned findings on subregional alterations and sex differences in alcohol dependence are yet to be corroborated in humans. Smaller volumes of the whole amygdala ${ }^{28-30}$ and hippocampus ${ }^{31-35}$ have been identified in people with alcohol dependence compared to controls. However, the neuroimaging evidence to date in people with alcohol dependence has focussed on mostly male samples and tends not to examine or report interactions with $\operatorname{sex}^{11}$. Preliminary evidence of sex-related effects in alcohol dependence show smaller total grey and white matter volumes $^{3,36,37}$ and smaller total volumes of the hippocampus $^{34}$ in alcohol-dependent females compared to male counterparts, despite equal or lesser alcohol use. There is also evidence of volume loss within specific subregions of the amygdala (i.e., basolateral) and the hippocampus (i.e., subiculum) in alcohol dependence ${ }^{38}$. However, whether such subregional effects are driven by either males or females, or by each sex equally, has not been examined. Methodological limitations may account for this knowledge gap, such as the use of small sample sizes with insufficient power to concurrently examine the effects of sex and alcohol on brain volumes, and the limited resolution and accuracy of traditional neuroimaging data analysis methods to segment amygdala and hippocampus subregions ${ }^{39}$. Global consortia comprising large population-representative samples, together with the recent release of new and reliable algorithms to segment subregions ${ }^{40}$, provide a unique opportunity to examine in detail amygdala and hippocampus subregions in males and females with alcohol dependence.

We aimed to validate animal findings in a sample of 966 human participants selected from the ENIGMA Addiction Working Group (https://www.enigmaaddictionconsortium. $\mathrm{com} /$ ). The sample comprised 323 people without alcohol dependence (225 males and 98 females) and a large sample of 643 people with alcohol dependence (418 males and 225 females). We hypothesised that: (i) on average, people with alcohol dependence versus controls would show smaller volumes of specific amygdala nuclei (i.e., central and basolateral) $)^{19-22}$ and hippocampal subfields (i.e., CA1, CA3, dentate gyrus and subiculum $)^{27-32}$; (ii) females with alcohol dependence would have smaller volumes than female controls and males with alcohol dependence $3,34,36,37,41-44$; (iii) a greater number of monthly standard alcohol drinks would predict lower volumes in the hypothesised subregions in both sexes ${ }^{3,36,37}$. Given the opportunities afforded by the large sample of this study, we also explored group and group-by-sex differences in additional amygdala nuclei (i.e., anterior amygdala area, corticoamygdaloid transition area, paralaminar, accessory basal, medial, and cortical nuclei) and hippocampal subfields (i.e., CA4, hippocampal-amygdaloid transition area, fimbria, fissure, tail, presubiculum, and parasubiculum).

\section{Materials and methods \\ Participants}

This study was pre-registered on the Open Science Framework in September 2018 (see https://osf.io/gz96w). 
Demographic, neuroimaging, and substance use (alcohol and tobacco) data were collected for 1,325 participants from 10 research sites that are part of the ENIGMA Addiction Working Group (https://www.enigmaaddictionconsortium. $\mathrm{com} /$ ). Supplementary Table 1 provides summary information for each site: including site location, study inclusion/ exclusion criteria, MRI scanner type, acquisition sequences and instruments used to measure substance use. We screened the original sample using the following exclusion criteria: (i) lifetime and/or current primary psychiatric disorders other than alcohol dependence and/or current dependence on substances other than alcohol $(n=125$ alcohol group); (ii) abstinence $>30$ days $(n=15$ alcohol group); (iii) IQ $<80$ ( $n=15$ alcohol group); (iv) missing data for key variables including sex ( $n=68$ alcohol group) and years of education ( $n=45$ alcohol group and $n=73$ controls); and (v) MRI artefacts that undermined the validity of the volumes ( $n=15$ alcohol group, and $n=3$ controls). The final sample comprised 966 participants aged on average 32.47 years $(S D=10.50$; Table 1$)$ and included 643 people (225 females) with alcohol dependence and 323 controls (98 females). All sites had obtained ethical approval from local committees and written informed consent from all participants.

\section{Structural MRI data acquisition and processing}

Automated parcellation of subcortical regions using structural T1-weighted brain images was performed at all sites using the recon-all pipeline in FreeSurfer 5.3 (http:// sufrer.nmr.mgh.harvard.edu/ $)^{45,46}$. We then used new FreeSurfer 6.0 algorithms (implemented within the development version: devel-20180612) $)^{40,47}$ to segment the bilateral total amygdala and its nuclei (i.e., anterior amygdaloid, corticoamygdaloid transition area, lateral, basal, paralaminar, accessory basal, medial, central, and cortical) and the bilateral total hippocampus and its subfields (i.e., tail, subiculum, presubiculim, parasubiculum, CA1, CA3, CA4, fissure, granular cells layer of the dentate gyrus [GC-ML-DG], molecular layer, hippocampus-amygdala transition area [HATA] and fimbria). Volumes for the a priori hippocampus dentate gyrus and for the basolateral amygdala were obtained by summing up those of smaller subregional outputs from FreeSurfer (i.e., hippocampus GC-ML-DG and molecular layer; amygdala basal and lateral nuclei, respectively).

All imaging data underwent standardised quality checks to reduce variability across sites, detect outliers, and invalid data points (http://enigma.ini.usc.edu/protocols/imagingprotocols). Additional stringent quality checks were run and entailed visual inspection of all hippocampal and amygdala subregions (see acknowledgments) using standardised protocols in collaboration with ENIGMA-MDD hippocampal subfields project which were adapted to apply to the new FreeSurfer algorithm (https://osf.io/b3uhw/).

\section{Statistical Analysis}

Chi-squared tests were run to test sex differences between groups. Linear mixed models were performed to examine group, sex, and group-by-sex differences for years of age, years of education, number of monthly standard alcohol drinks ${ }^{1}$, number of monthly cigarettes, and brain volumes. Linear mixed models were chosen as they statistically accommodate dependency between observations (i.e., data points) in nested designs where data are collected from distinct research sites ${ }^{48}$. Site was treated as a random effect to account for the systematic site-level variation in the dependent variables expected to occur from differences in MRI scanner types and sequences, imaging, and behavioural testing protocols. Intra-class correlation (ICC) measured the extent of variation explained by site-level differences ${ }^{48}$.

First, we examined effects of group, sex, and group-bysex interactions on the volumes of a priori amygdala volumes (total, and basolateral, and central nuclei) and hippocampus volumes (total, CA1, CA3, dentate gyrus, and subiculum subfields), adjusting for major confounders (age, education, and intracranial volume). Sensitivity analyses using tobacco exposure as a covariate (number of cigarettes per month), were further run on a subsample where information on these variables was also available (488 people with alcohol dependence and 140 controls).

Second, we measured if monthly standard drinks predicted a priori amygdala volumes (total, and basolateral, and central nuclei) and hippocampus volumes (total, and dentate gyrus, CA1, and CA3 subfields), separately in males and females with alcohol dependence, accounting for age, education, and intracranial volume. We assured that the assumptions for linear mixed models were met, including normal distribution of random effects. Monthly standard alcohol drinks were square root transformed as they showed positive skewness (i.e., 2.03 before and 1.00 after transformation).

All statistical models described above were also run in exploratory tests on the volumes of additional amygdalar nuclei (i.e., accessory basal, anterior, cortical, corticoamygdaloid transition area, medial, paralaminar), hippocampal subfields (i.e., CA4, fimbria, fissure, HATA, parasubiculum, presubiculum, tail), and total cortical grey matter, white matter, and cerebrospinal fluid volumes.

A false discovery rate (FDR)-corrected statistical threshold of $p(\mathrm{FDR})<0.05$ was used to control for multiple comparisons ${ }^{49}$ and was applied separately for a priori and exploratory analyses. Significant main or interaction effects were interrogated with pairwise comparisons

\footnotetext{
Standard drinks were defined according to the National Institute on Alcohol Abuse and Alcoholism (NIAAA) criteria (https://www.niaaa.nih.gov/whatstandard-drink).
} 


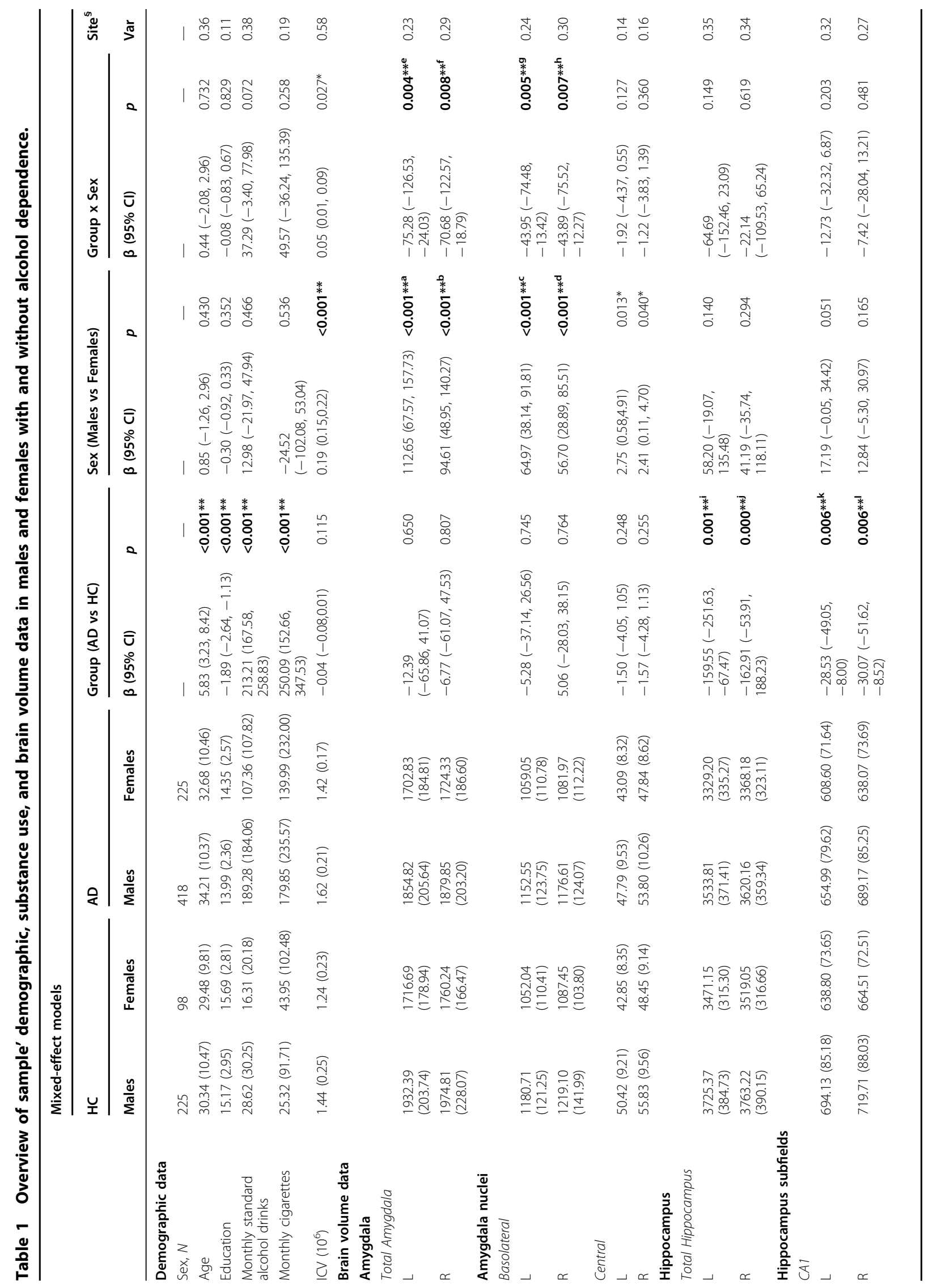




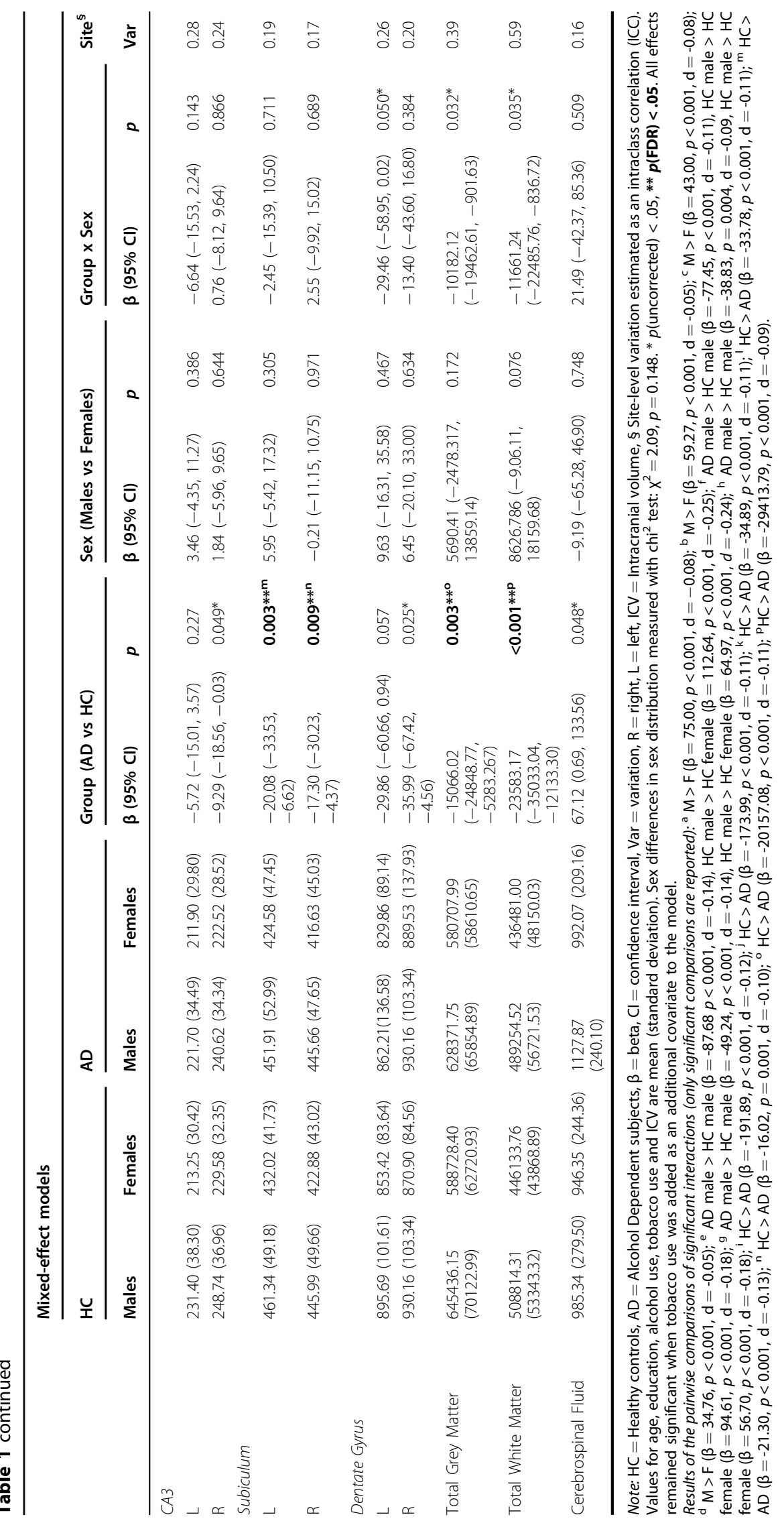


between the groups and/or sexes. In the text, we express the percentage (\%) calculated as a \% difference between the two observed means. Effect sizes were estimated for the significant $p(\mathrm{FDR})<0.05$ group and group-by-sex effects using Cohen's $d$ and based on the marginal means predicted by the model. All statistical analyses were performed using STATA 15.1 (StataCorp; 2017).

\section{Results}

Basic demographic information and brain volume data are provided in Table 1. The alcohol-dependent and control groups were matched by sex. The group comprising people with alcohol dependence was older, had fewer years of education, and had higher monthly alcohol standard drinks and cigarettes than the control group. Males in both the alcohol-dependent and control groups consumed more monthly standard alcohol drinks than females. The alcohol-dependent group also had significantly smaller total grey and white matter volumes than healthy controls.

\section{Amygdala volumes \\ Group, sex, and group-by-sex effects on the volumes of the amygdala and its nuclei}

In the analysis of a priori amygdala volumes across group, sex, and group-by-sex (Table 1), there were no main effects of group, but there were significant main effects of sex and group-by-sex interactions for the bilateral total and basolateral amygdala (Table 1; accounting for age, education, intracranial volume, and tobacco use). Pairwise comparisons showed that alcoholdependent males had significantly lower volumes of the total amygdala (5\% smaller) and basolateral nucleus (3\% smaller) than male controls (Fig. 1). There were no significant differences between females with alcohol dependence and female controls within the a priori amygdala nuclei. There were no significant group or group-by-sex effects within the central amygdala.

Within select exploratory amygdala nuclei (i.e., accessory basal, anterior and cortico-amygdaloid transition; Table 2 and Fig. 2) there were significant main effects of sex (males larger than females) and significant group-bysex interactions, whereby males (but not females) with alcohol dependence had significantly smaller volumes than male controls.

\section{Association between alcohol dosage and amygdala volumes in males and females}

More monthly standard alcohol drinks predicted smaller total and basolateral amygdala volume in males, but not in females, with alcohol dependence (Fig. 1); after accounting for age, education, and intracranial volume. In females, there was a significant negative association between monthly standard alcohol drinks and volumes of the left central nucleus.

\section{Hippocampus volumes \\ Group, sex, and group-by-sex effects on the volumes of the hippocampus and its subfields}

In the analysis of the a priori hippocampus regions, there were significant main effects of group within the bilateral total hippocampus and select subfields (CA1 and subiculum; Table 1), accounting for age, education, intracranial volume, and tobacco use. Pairwise analyses demonstrated 5\% smaller volumes of the total hippocampus, $5 \%$ smaller CA1, and $3 \%$ smaller subiculum in the alcohol-dependent versus control group (Fig. 3). Specifically, the relative smaller volumes in males with alcohol dependence compared to male controls was comparable to that observed in alcohol-dependent females versus control females (e.g., 6\% difference between males and $5 \%$ difference in females in the left hippocampus $[p$ 's $<0.001])$. We did not detect significant main effects of sex or interactions between group and sex for any a priori hippocampus subfields.

Within the exploratory subfields, there were significant main effects of group (Table 2. The alcohol dependent compared with the control group had significantly lower volumes of the right CA4 (3\% smaller) and bilateral hippocampal tail ( $5 \%$ smaller); however, the significance of these effects dissipated when tobacco use was added as a covariate to these models. Within the exploratory HATA and fimbria hippocampal subfields, there were significant main effects of sex (males larger than females), and significant group-by-sex interactions whereby males with alcohol dependence compared to male controls had significantly lower bilateral HATA ( $11 \%$ smaller) and right fimbria ( $8 \%$ smaller) volumes, after accounting for age, education, ICV, and tobacco use (Table 2 and Fig. 2).

\section{Association between alcohol dosage and hippocampus volumes in males and females}

There was no association between monthly standard alcohol drinks and any of the hippocampal volumes in males or females.

\section{Sensitivity analysis on a priori amygdalar and hippocampal total and subregional volumes}

In the alcohol-dependent group, males consumed significantly more monthly standard alcohol drinks than females (Table 1). Thus, we could not resolve whether dose-related lower volumes of the total and basolateral amygdala in males were driven by the higher drinking levels in males than females. To address this issue, we performed a sensitivity analysis (Supplementary Table 2). First, we matched the number of monthly standard drinks between men and women with alcohol dependence 

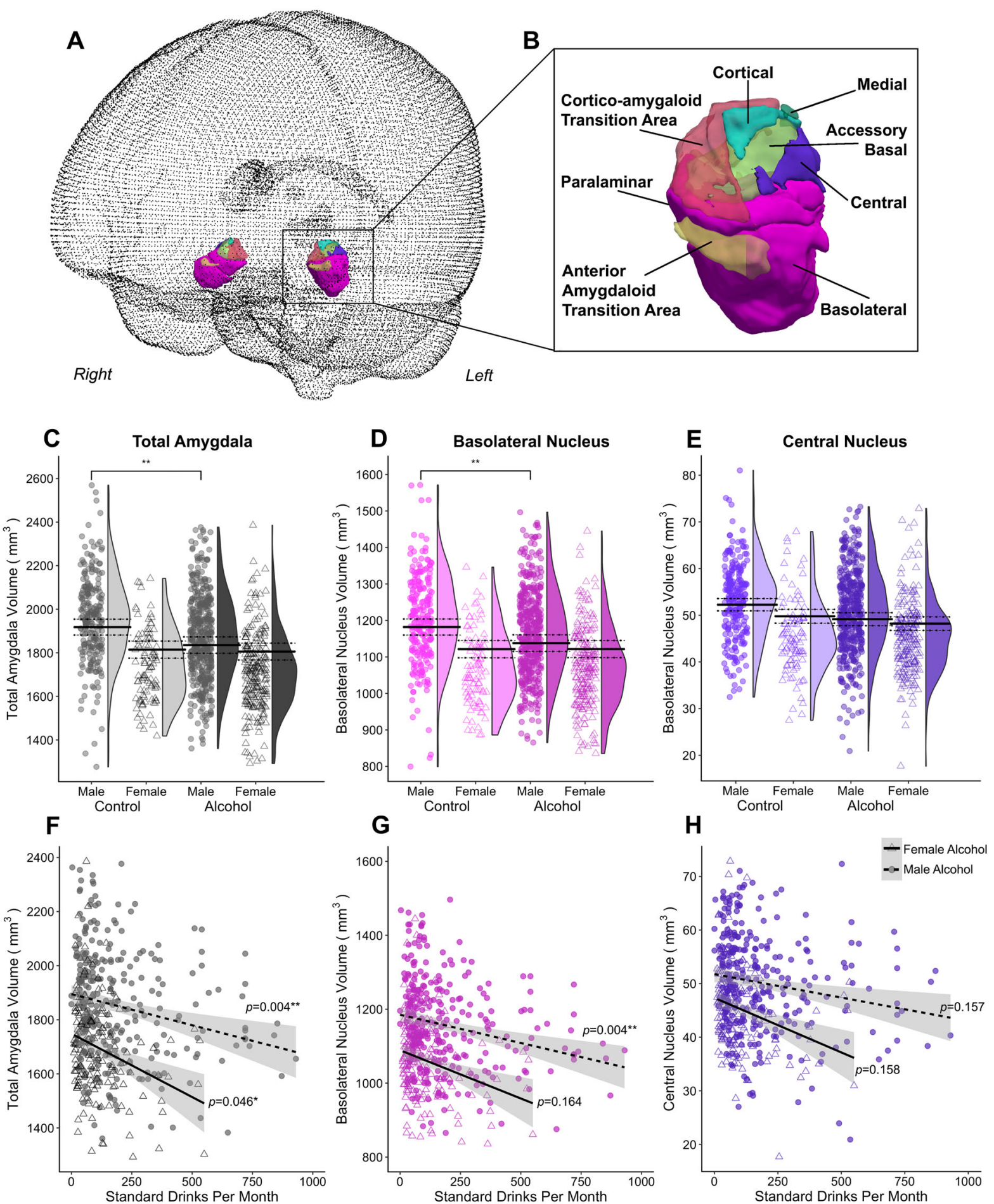

Fig. 1 Overview of group by sex effects on the volume of a-priori amygdala region and amygdala nuclei. A Overview of glass brain section showing $3 \mathrm{D}$ rendering and $\mathbf{B}$ close up of $3 \mathrm{D}$ renders of all amygdala nuclei from an example participant. Plots of the a priori amygdala regions, comprising $\mathbf{C}$ total amygdala in greyscale, $\mathbf{D}$ basolateral nuclei in pink, and $\mathbf{E}$ central nuclei in purple containing individual data points and the probability density of the data stratified by group and sex (the group average of the estimated marginal means predicted by the models are indicated by the solid black line, with dotted lines indicating the standard error of the marginal means). The bottom panel shows regression plots for volume of the $\mathbf{F}$ total amygdala, $\mathbf{G}$ basolateral nuclei, and $\mathbf{H}$ central nuclei by alcohol use (standard alcohol drinks per month) in male and female alcohol-dependent participants, adjusted for intracranial volume, age, and education. Only the association in the male group for the total and basolateral amygdala remained significant after FDR correction. The left and right hemispheres for the nuclei have been collapsed. ${ }^{* *} p($ FDR $)<05$. 


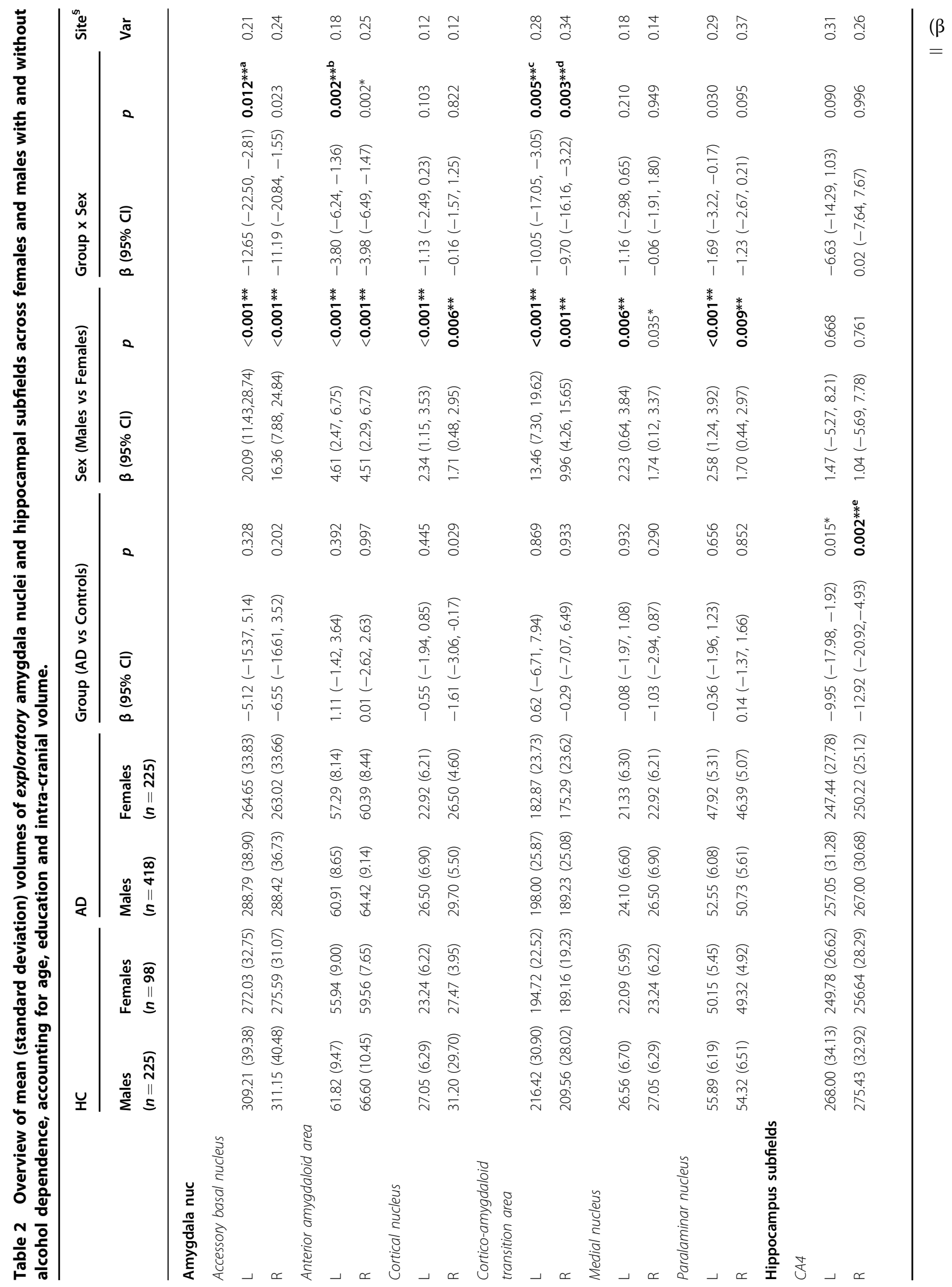




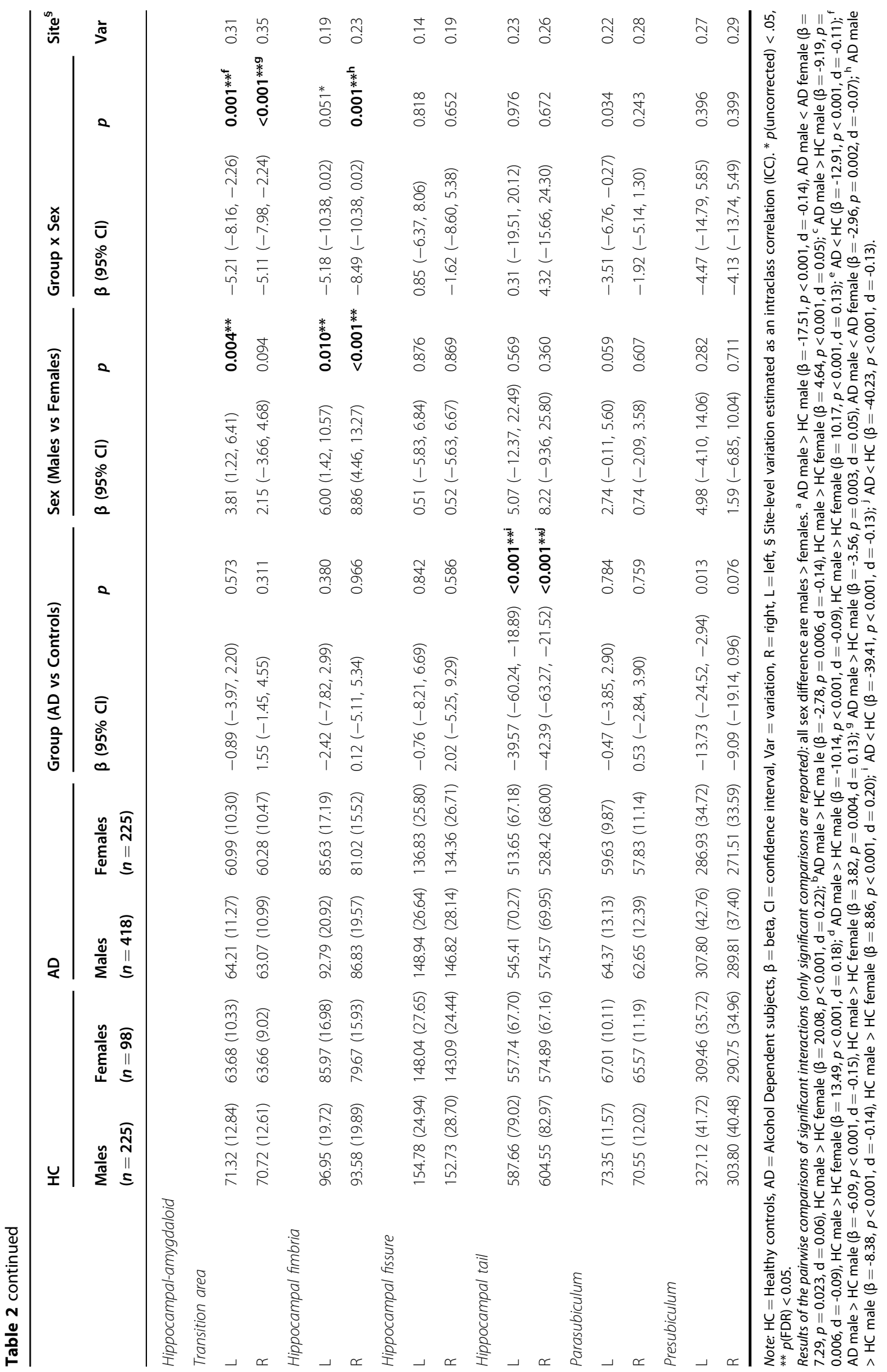


Amygdala Nuclei, Exploratory Analysis
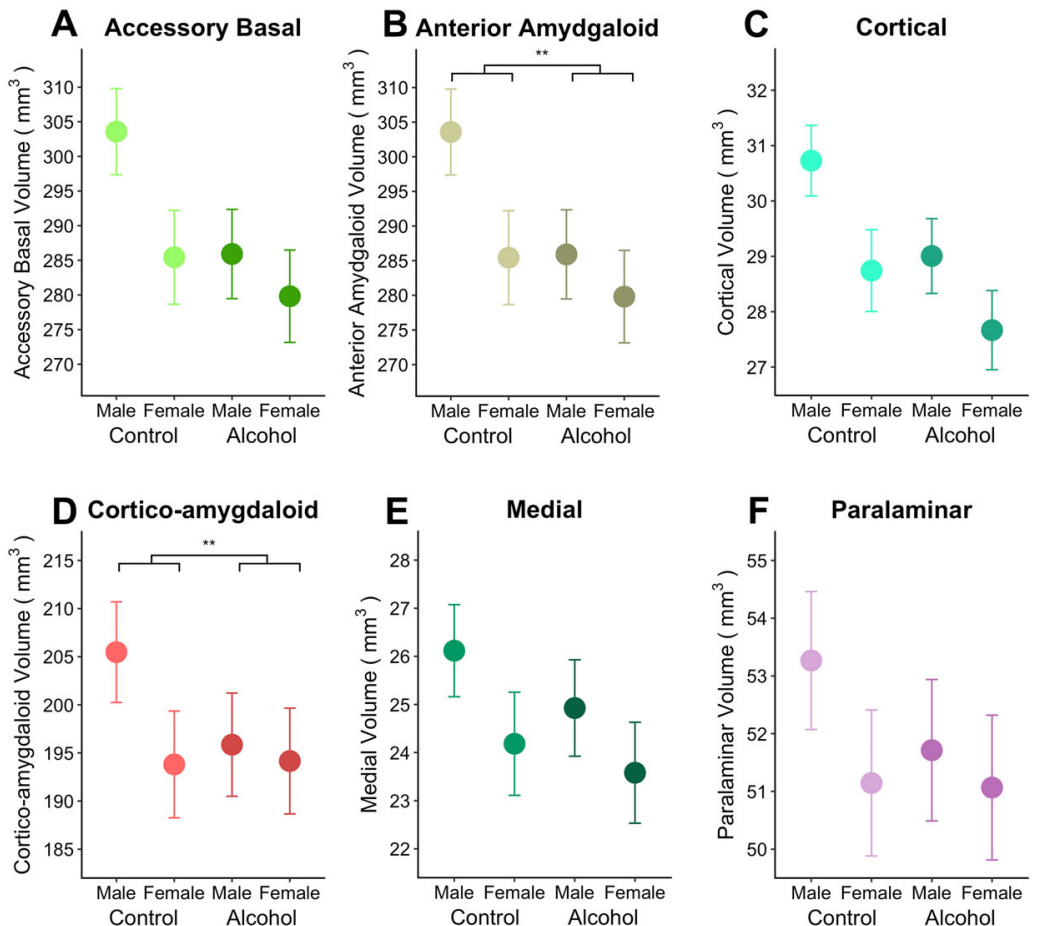

Hippocampus Subfields, Exploratory Analysis
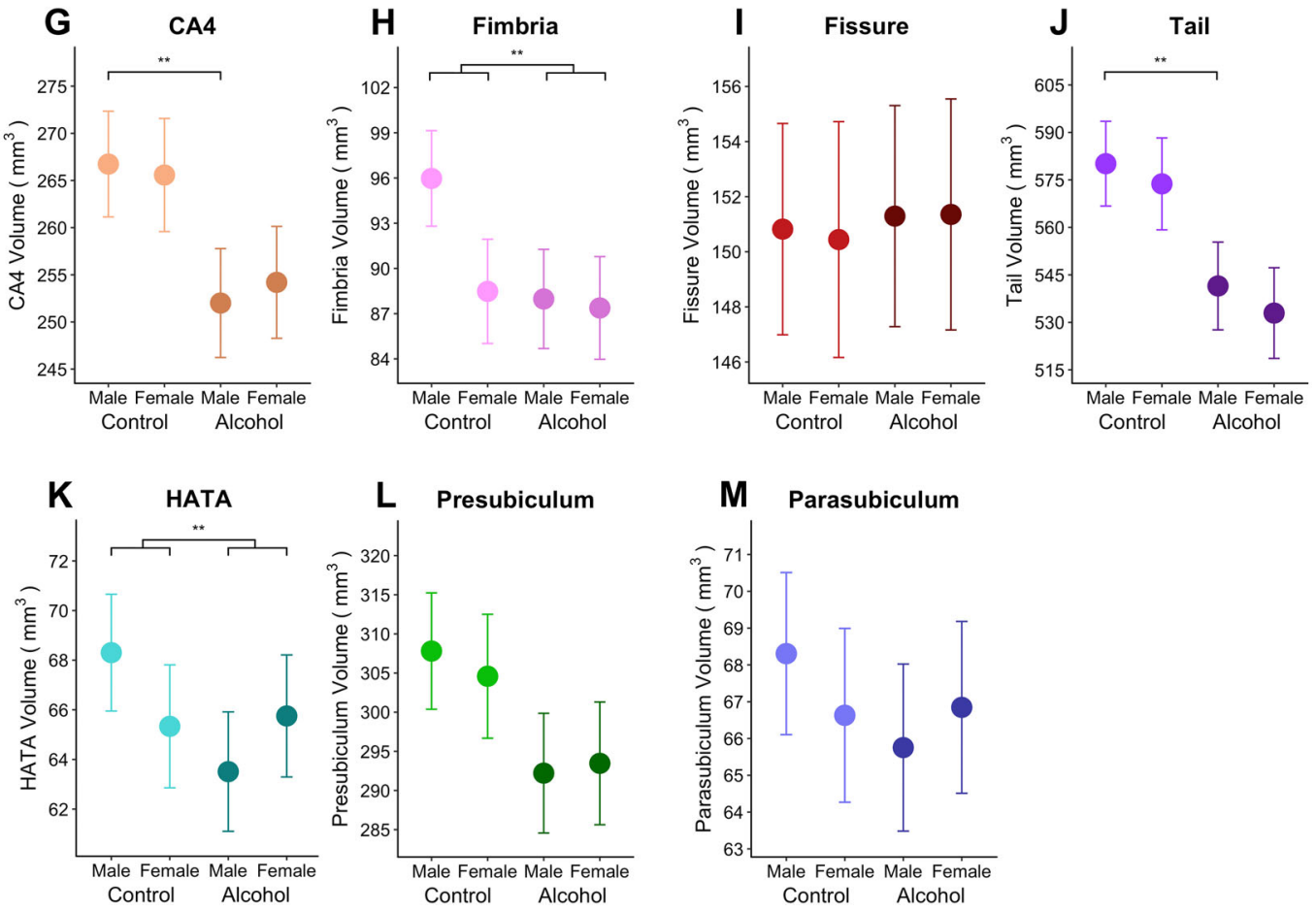

Fig. 2 Overview of group by sex effects on the volume of exploratory amygdala nuclei and hippocampus subfields. Plots showing the estimated marginal means predicted by the model for the exploratory amygdala nuclei $\mathbf{A}$ accessory basal nucleus, $\mathbf{B}$ anterior amygdaloid transition area, $\mathbf{C}$ cortical nucleus, D cortico-amygdaloid transition area, $\mathbf{E}$ medial nucleus, and $\mathbf{F}$ paralaminar nucleus; and hippocampus subfields: $\mathbf{G}$ cornu ammonis 4 (CA4), $\mathbf{H}$ fimbria, I fissure, $\mathbf{J}$ tail, $\mathbf{K}$ hippocampal-amygdaloid tranisiton area (HATA), L presubiculum and $\mathbf{M}$ parasubiculum. The left and right hemispheres for the nuclei have been collapsed. Error bars represent the standard error of the marginal mean. ${ }^{* *} p(\mathrm{FDR})<0.05$. 

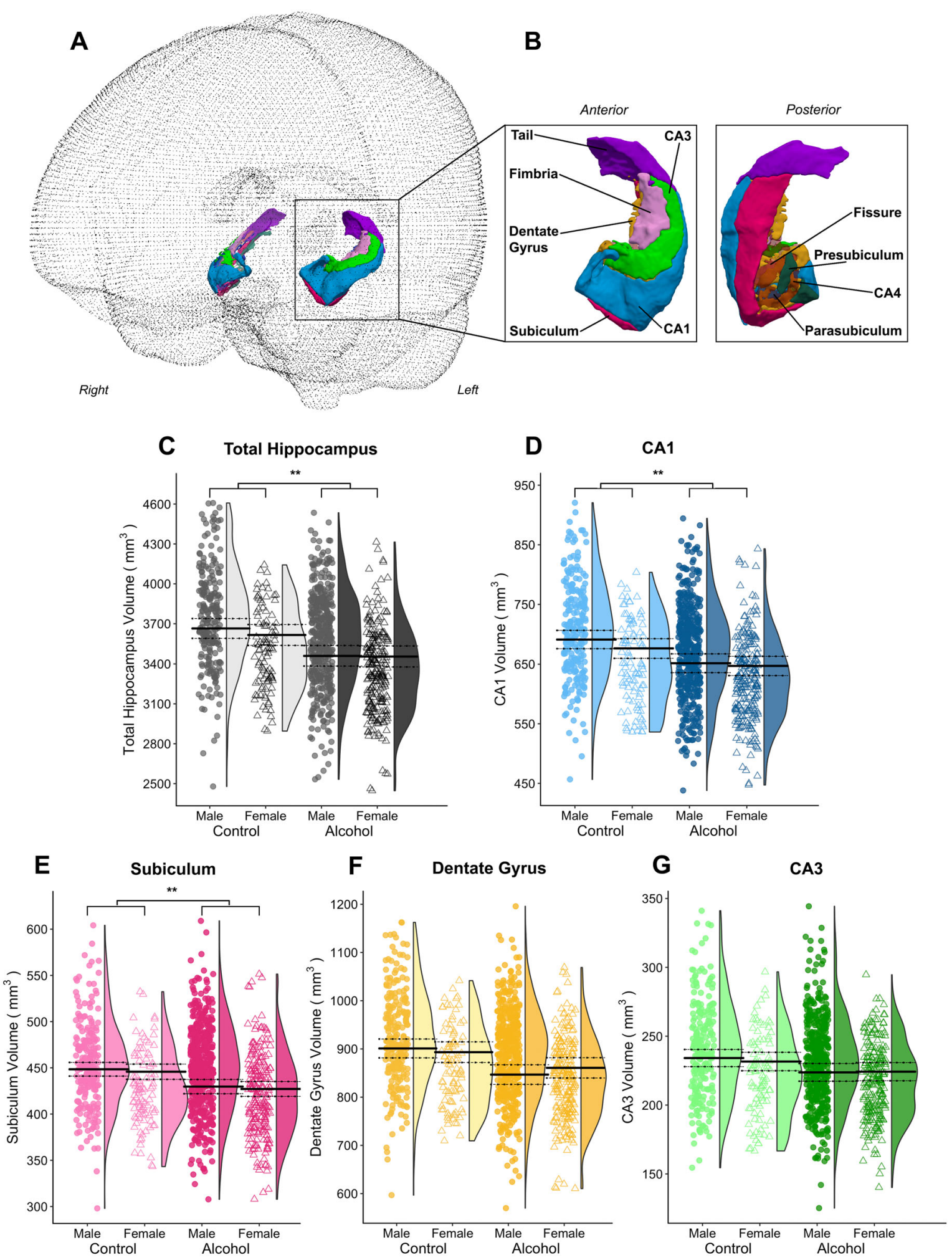

Fig. 3 Overview of group by sex effects on the volume of a-priori hippocampual region and hippocampus subfields. A Overview of glass brain section showing $3 \mathrm{D}$ rendering and $\mathbf{B}$ close up of $3 \mathrm{D}$ renders of all hippocampal subfields from an example participant. Plots of the a priori C total hippocampus and $\mathbf{D}$ cornu ammonis 1 (CA1), E subiculum, $\mathbf{F}$ dentate gyrus, and $\mathbf{G}$ cornu ammonis 3 (CA3) subfields; containing individual data points and the probability density of the data stratified by group and sex (the group average of the estimated marginal means predicted by the models are indicated by the solid black line, with dotted lines indicating the standard error of the marginal means). The left and right hemispheres for the subfields have been collapsed. ${ }^{* *} p(F D R)<.05$. 
$0.53,95 \% C I=[-0.15,1.21], p=0.129)$ via removing alcohol-dependent males with the highest drinking levels ( $n=186$ males who consumed $<280$ monthly standard alcohol drinks). Then, we re-ran the statistical models that examined effects of group, sex, and group-by-sex on the volumes of a priori amygdalar and hippocampal volumes, and the association between alcohol dose and volumes in this sub-sample. The result of these analyses corroborated that main findings of a significant group-by-sex interaction within total and basolateral amygdala volumes (Supplementary Table 2). The negative interaction between monthly standard alcohol drinks and right total amygdala volume in males with alcohol dependence was also corroborated $(\beta=-9.68,95 \% C I=[-16.67,-2.70]$, $p=0.007$ ); however, the negative interactions between monthly standard alcohol drinks and volume of the left total amygdala or bilateral basolateral amygdala nuclei were not evident in the sensitivity subsample.

\section{Discussion}

In this study, we demonstrated that alcohol dependence is associated with alterations of select subregions of the hippocampus and amygdala, some of which were similar and distinct between sexes. Our key findings were that males (but not females) with alcohol dependence had dosedependent smaller volumes of the basolateral amygdala nucleus, and smaller volumes in exploratory subregions of the amygdala and hippocampus (i.e., cortico-amygdaloid transition, anterior, accessory basal, HATA, and fimbria). Additionally, alcohol dependence was associated with smaller volumes of total brain estimates of grey and white matter and within select hippocampal subfields (i.e., CA1, CA4, and subiculum), equally in men and women.

We report sex differences in the volumes of the total amygdala, select amygdala nuclei (i.e., basolateral, anterior amygdaloid area, accessory basal), and hippocampal subfields that connect with the amygdala (i.e., corticoamygdaloid transition area and HATA) ${ }^{50}$. Our findings are partially consistent with those from previous work on alcohol dependence (i.e., smaller total amygdala and basolateral nucleus) where sex differences were not examined ${ }^{28-30,38}$. Lower amygdala volumes affected select amygdala nuclei that are implicated in behaviours characteristic of alcohol dependence, such as greater alcohol seeking ${ }^{51}$, higher alcohol craving and relapse risk ${ }^{38}$, and stress ${ }^{52}$ (i.e., anterior amygdaloid area, accessory basal nucleus). Thus, one could speculate that smaller volumes of select amygdala nuclei predate alcohol dependence in males. This may help explain why males are twice as likely to have alcohol dependence compared to females ${ }^{28,30,38}$. Alternatively, dose-dependent smaller volumes of the amygdala's basolateral nucleus might reflect neuronal loss (e.g., apoptosis) due to the neurotoxic effects from the chronic exposure to ethanol, that is likely to occur in people with alcohol dependence ${ }^{53}$.
Within the hippocampus, we did not identify the predicted interactions between alcohol dependence and sex. We did, however, support our hypothesis and previous findings that people with alcohol dependence compared to controls have smaller hippocampal total volume ${ }^{31-35}$. This alteration may represent a shared neurobiological correlate as it was apparent in both males and females with alcohol dependence. We also replicated previous evidence that smaller total hippocampus volumes were driven by smaller volumes of select hippocampus subfields, including the CA1, CA4, and subiculum ${ }^{38}$. Lower hippocampal subfield volumes may reflect neuronal cell loss resulting from the neurotoxic effects of chronic alcohol exposure ${ }^{20,22,23}$, reduced hippocampal neurogenesis $^{17}$, or represent a shared neurobiological vulnerability that predates alcohol dependence in both men and women. Volumetric reduction within the hippocampus may have implications for aberrant learning and memory processes characteristic of alcohol dependence ${ }^{54}$, particularly within the CA1 subfield which is implicated in memory retrieval and consolidation ${ }^{27}$.

We confirmed previous findings that alcohol dependence is associated with smaller global estimates of brain volume (grey and white matter) 37,55 . However, we failed to find any global or regional volumetric alterations specific to females with alcohol dependence. This finding supports evidence that a telescoping effect of alcohol dependence in females is not evident within studies of the general population ${ }^{56}$; however, it contrasted with our predictions and considerable evidence that females are more vulnerable to the neurotoxic effects of alcohol ${ }^{3,37,44}$. Sensitivity analyses corroborated our results in subsamples where males and females were matched by monthly standard drinks, and when analyses were re-run in males and females separately. We cannot determine whether lower amygdala volumes observed in alcohol-dependent males but not females (compared to their control counterparts) reflects either a (partly) distinct pathophysiology of alcohol dependence in different sexes, or phenotype differences in our sample that may have caused smaller amygdala nuclei volumes in males, but not in females, with alcohol dependence. For example, the structural alterations to the amygdala observed in males (but not in females) with alcohol dependence compared to controls, may have been caused by variables such as a distinct history of alcohol and other substance use, sex hormones, sleep quality, negative life events, and/or stress that differ between males and females ${ }^{5}$, that were not available in this sample. Likewise, a possible explanation for null results in females with alcohol dependence compared to female controls, is that only a subgroup of women with a high level of stress may be vulnerable to developing alterations of select amygdala and hippocampus subregions, as shown in animal models ${ }^{14,57}$. Indeed, our 
sample may have had heterogeneous levels of stress and related factors, as has been reported in previously examined samples of women with alcohol dependence (e.g., early life trauma, abuse $)^{14}$. However, we cannot relate our data to stress levels as these were not available.

\section{Limitations}

This study was cross-sectional, and we cannot exclude the possibility that the smaller volumes observed predated the onset of alcohol dependence. Longitudinal studies will be necessary to track whether alterations to hippocampus and amygdala subregional structure reflect trajectories before or during alcohol dependence or following its recovery. We had no data on mental health symptoms and limited data on substance use due to the heterogeneous testing protocols between sites. However, this was mitigated by screening for psychiatric comorbidities and controlling for the role of tobacco use and education. In addition, the new FreeSurfer segmentation algorithm has not been extensively validated; in response, we performed extensive visual quality assurance of all images (https://osf.io/wu78p/). A further strength of our study was that the large aggregated sample allowed adequate power to detect subtle effects of alcohol dependence and its interaction with sex.

\section{Conclusions}

Alcohol dependence was associated with lower volumes within select hippocampus subfields in both males and females and within distinct amygdala nuclei in males only and in a dose-dependent fashion. Thus, we believe the systematic assessment of sex differences in alcohol dependence is warranted, using a subregion-specific approach and longitudinal designs, to track over time the neurobiological mechanisms that predate and follow alcohol dependence' onset, recovery, and relapse in both males and females. This knowledge will be crucial to advance current neuroscientific theories of addiction and to ultimately inform personalised treatment targets.

\begin{abstract}
Acknowledgements
We thank Diny Thomson, Danielle Tichelaar, Emily Robinson, Emily Watt, Iris Starcheus, Jamie Gladwell, John Tzaferis, Kirsty Kearney, and Simone Mizzi for their significant contributions to the image quality checks. We thank Preveetha Patalay for her helpful contributions to the statistical modelling; Philip Saemann for providing comments on code to generate the images for visual inspection; and Juan Dominguez for his helpful comments on the code to perform the FreeSurfer segmentation. This work was supported by the MASSIVE HPC facility (www.massive.org.au). This work was also supported by funding from the U.S. National Institute of Health (NIH R01DA047119) and the
\end{abstract} U.S. National Institute of Mental Health (NIH/NIMH R01MH116147).

\section{Author details}

${ }^{1}$ Neuroscience of Addiction \& Mental Health Program, Healthy Brain and Mind Research Centre, School of Behavioural \& Health Sciences, Faculty of Health Sciences, Australian Catholic University, Melbourne, VIC, Australia. ${ }^{2}$ Department of Neurosciences, Biomedicine and Movement Sciences, Section of Psychiatry, University of Verona, Verona, Italy. ${ }^{3}$ Department of Neurosciences and Mental
Health, Fondazione IRCCS Ca' Granda Ospedale Maggiore Policlinico, Milan, Italy. ${ }^{4}$ Department of Psychology, University of Oregon, Eugene, OR, USA. ${ }^{5}$ Department of Psychiatry, UMC Utrecht Brain Center, Utrecht University, Utrecht, Netherlands. 'Department of Pathophysiology and Transplantation, University of Milan, Milan, Italy. ${ }^{7}$ Turner Institute for Brain and Mental Health, School of Psychological Sciences, Monash University, Clayton, VIC, Australia. ${ }^{8}$ Department of Psychology, University of Amsterdam, Amsterdam, the Netherlands. ${ }^{9}$ Department of Psychiatry, Amsterdam Institute for Addiction Research and Amsterdam Neuroscience Research Institute, Amsterdam UMC, University of Amsterdam, Amsterdam, the Netherlands. ${ }^{10}$ School of Psychological Sciences, University of Melbourne, Melbourne, Australia. ${ }^{11}$ Department of Psychology and Neuroscience, University of Colorado Boulder, Boulder, CO, USA. ${ }^{12}$ Clinical Neurolmaging Research Core, Office of the Clinical Director, National Institute on Alcohol Abuse and Alcoholism, Bethesda, MD, USA. ${ }^{13}$ Department of Neurosciences and Behavior, Ribeirão Preto Medical School, University of São Paulo, Av. Bandeirantes, Ribeirão Preto, São Paulo, Brazil. ${ }^{14}$ School of Psychology and Illawarra Health and Medical Research Institute, University of Wollongong, Wollongong, Australia.

${ }^{15}$ Department of Psychiatry, Yale University School of Medicine, New Haven, CT, USA. ${ }^{16}$ Orygen, The National Centre of Excellence in Youth Mental Health, Parkville, Australia. ${ }^{17}$ Centre for Youth Mental Health, The University of Melbourne, Melbourne, VIC 3052, Australia. ${ }^{18}$ Institute of Psychology. Cognitive Psychology Unit, Leiden University, Leiden, The Netherlands. ${ }^{19}$ Departments of Neurology, Radiology, Engineering, \& Psychiatry, Imaging Genetics Center, Mark \& Mary Stevens Institute for Neuroimaging \& Informatics, Keck School of Medicine, University of Southern California, Marina del Rey, CA, USA.

${ }^{20}$ Department of Psychiatry, Université de Montreal, CHU Ste Justine Hospital, Montreal, QC, Canada. ${ }^{21}$ Departments of Psychiatry and Psychology, University of Vermont, Burlington, VT, USA

\section{Author contributions}

Sally Grace and Valentina Lorenzetti wrote the first draft and prepared all drafts for submission; Maria Gloria Rosetti, Yann Chye, Sally Grace, and Valentina Lorenzetti conceived the analyses, Maria Gloria Rosetti screened the data set for exclusion criteria; Sally Grace conducted analyses; Chao Suo provided technical support for analyses; Valentina Lorenzetti supervised all analyses; Nicolas Allen, Janna Cousijn, Hugh Garavan, Anneke Goudriaan, Robert Hester, Reza Momenan, Rocio Martin-Santos, Nadia Solowij, Lianne Schamaal, Zsuzika Sjoerds, Ruth van Holst, Dick Veltman, Murat Yücel, and the ENIGMA Addiction Working Group facilitated data collection and prepared individual datasets and conducted primary analyses and image quality checks; Izelle Labuschagne, Peter Rendell, and Gill Terrett provided resource support. All authors reviewed the manuscript and approved it for submission.

\section{Data availability}

The datasets generated during and/or analysed during the current study are available from the corresponding author on reasonable request.

\section{Conflict of interest}

Paul Thomson received grant support from Biogen, Inc., for work unrelated to this manuscript.

\section{Publisher's note}

Springer Nature remains neutral with regard to jurisdictional claims in published maps and institutional affiliations.

Supplementary information The online version contains supplementary material available at https://doi.org/10.1038/s41398-021-01204-1.

Received: 30 April 2020 Revised: 28 September 2020 Accepted: 26 October 2020

Published online: 04 March 2021

\section{References}

1. World Health Organization. Global status report on alcohol and health, 2018. World Health Organization 2018. 
2. Rehm, J. et al. Alcohol and Global Health 1 Global burden of disease and injury and economic cost attributable to alcohol use and alcohol-use disorders. Lancet 373, 2223-2233 (2009).

3. Mann, K. et al. Neuroimaging of gender differences in alcohol dependence: are women more vulnerable? Alcohol.: Clin. Exp. Res. 29, 896-901 (2005).

4. Slade, T. et al. Birth cohort trends in the global epidemiology of alcohol use and alcohol-related harms in men and women: systematic review and metaregression. Br. Med. J. Open 6, e011827 (2016).

5. Eagon, P. K. Alcoholic liver injury: influence of gender and hormones. World J. Gastroenterol. 16, 1377-1384 (2010).

6. Hernandez-Avila, C. A., Rounsaville, B. J. \& Kranzler, H. R. Opioid-, cannabis- and alcohol-dependent women show more rapid progression to substance abuse treatment. Drug Alcohol Depend. 74, 265-272 (2004).

7. Boykoff, N. et al. Gender differences in the relationship between depressive symptoms and cravings in alcoholism. Am. J. Addictions 19, 352-356 (2010).

8. Westermeyer, J. \& Boedicker, A. E. Course, severity, and treatment of substance abuse among women versus men. Am. J. Drug Alcohol Abus. 26, 523-535 (2000).

9. Wassum, K. M. \& Izquierdo, A. The basolateral amygdala in reward learning and addiction. Neurosci. Biobehav. Rev. 57, 271-283 (2015).

10. Buhler, M. \& Mann, K. Alcohol and the human brain: a systematic review of different neuroimaging methods. Alcohol.: Clin. Exp. Res. 35, 1771-1793 (2011).

11. Lind, K. E. et al. Sex disparities in substance abuse research: evaluating 23 years of structural neuroimaging studies. Drug Alcohol Depend. 173, $92-98$ (2017).

12. Clayton, J. A. \& Collins, F. S. Policy: NIH to balance sex in cell and animal studies. Nature 509, 282-283 (2014).

13. Koob, G. F. \& Volkow, N. D. Neurobiology of addiction: a neurocircuitry analysis. Lancet Psychiatry 3, 760-773 (2016).

14. Logrip, M. L., Oleata, C. \& Roberto, M. Sex differences in responses of the basolateral-central amygdala circuit to alcohol, corticosterone and their interaction. Neuropharmacology 114, 123-134 (2017).

15. Meil, W. M. \& See, R. E. Lesions of the basolateral amygdala abolish the ability of drug associated cues to reinstate responding during withdrawal from selfadministered cocaine. Behav. Brain Res. 87, 139-148 (1997).

16. Koob, G. F. The role of the striatopallidal and extended amygdala systems in drug addiction. Ann. N. Y. Acad. Sci. 877, 445-460 (1999).

17. Ji, Z. et al. Binge alcohol exposure causes neurobehavioral deficits and GSK3beta activation in the hippocampus of adolescent rats. Sci. Rep. 8, 3088 (2018).

18. Miki, T., Harris, S. J., Wilce, P. A., Takeuchi, Y. \& Bedi, K. S. Effects of age and alcohol exposure during early life on pyramidal cell numbers in the CA1-CA3 region of the rat hippocampus. Hippocampus 14, 124-134 (2004).

19. McMullen, P. A., Saint-Cyr, J. A. \& Carlen, P. L. Morphological alterations in rat CA1 hippocampal pyramidal cell dendrites resulting from chronic ethanol consumption and withdrawal. J. Comp. Neurol. 225, 111-118 (1984).

20. Mandyam, C. D. The interplay between the hippocampus and amygdala in regulating aberrant hippocampal neurogenesis during protracted abstinence from alcohol dependence. Front Psychiatry 4, 61 (2013).

21. Fujii, S., Yamazaki, Y., Sugihara, T. \& Wakabayashi, I. Acute and chronic ethanol exposure differentially affect induction of hippocampal LTP. Brain Res. 1211, 13-21 (2008).

22. Walls, S. A., Macklin, Z. L. \& Devaud, L. L. Ethanol-induced loss-of-righting response during ethanol withdrawal in male and female rats: associations with alterations in Arc labeling. Alcohol.: Clin. Exp. Res. 36, 234-241 (2012).

23. Zhou, L. et al. Fos expression induced by cocaine-conditioned cues in male and female rats. Brain Struct. Funct. 219, 1831-1840 (2014).

24. Maynard, M. E., Barton, E. A., Robinson, C. R., Wooden, J. I. \& Leasure, J. L. Sex differences in hippocampal damage, cognitive impairment, and trophic factor expression in an animal model of an alcohol use disorder. Brain Struct. Funct. 223, 195-210 (2018)

25. Matsumoto, N., Kitanishi, T. \& Mizuseki, K. The subiculum: Unique hippocampal hub and more. Neurosci. Res. 143, 1-12 (2019).

26. Suthana, N. A. et al. High-resolution $7 \mathrm{~T} \mathrm{fMRI}$ of Human Hippocampal Subfields during Associative Learning. J. Cogn. Neurosci. 27, 1194-1206 (2015).

27. La, C. et al. Hippocampal CA1 subfield predicts episodic memory impairment in Parkinson's disease. Neuroimage: Clin. 23, 101824 (2019).

28. Fein, G. et al. Brain atrophy in long-term abstinent alcoholics who demonstrate impairment on a simulated gambling task. Neuroimage 32, 1465-1471 (2006).
29. Benegal, V., Antony, G., Venkatasubramanian, G. \& Jayakumar, P. N. Imaging study: gray matter volume abnormalities and externalizing symptoms in subjects at high risk for alcohol dependence. Addiction Biol. 12, 122-132 (2007).

30. Beck, A. et al. Effect of brain structure, brain function, and brain connectivity on relapse in alcohol-dependent patients. Arch. Gen. Psychiatry 69, 842-852 (2012).

31. Beresford, T. P. et al. Hippocampus volume loss due to chronic heavy drinking. Alcohol.: Clin. Exp. Res. 30, 1866-1870 (2006)

32. Nagel, B. J., Schweinsburg, A. D., Phan, V. \& Tapert, S. F. Reduced hippocampal volume among adolescents with alcohol use disorders without psychiatric comorbidity. Psychiatry Res.: Neuroimaging 139, 181-190 (2005).

33. De Bellis, M. D. et al. Hippocampal volume in adolescent-onset alcohol use disorders. Am. J. Psychiatry 157, 737-744 (2000).

34. Agartz, I., Momenan, R., Rawlings, R. R., Kerich, M. J. \& Hommer, D. W. Hippocampal volume in patients with alcohol dependence. Arch. Gen. Psychiatry 56, 356-363 (1999).

35. Sullivan, E. V., Marsh, L., Mathalon, D. H., Lim, K. O. \& Pfefferbaum, A. Anterior hippocampal volume deficits in nonamnesic, aging chronic alcoholics. Alcohol.: Clin. Exp. Res. 19, 110-122 (1995)

36. Mann, K., Batra, A., Günthner, A. \& Schroth, G. Do women develop alcoholic brain damage more readily than men? Alcohol.: Clin. Exp. Res. 16, 1052-1056 (1992).

37. Hommer, D., Momenan, R., Kaiser, E. \& Rawlings, R. Evidence for a genderrelated effect of alcoholism on brain volumes. Am. J. Psychiatry 158, 198-204 (2001).

38. Wrase, J. et al. Amygdala volume associated with alcohol abuse relapse and craving. Am. J. Psychiatry 165, 1179-1184 (2008)

39. Wisse, L. E., Biessels, G. J. \& Geerlings, M. I. A critical appraisal of the hippocampal subfield segmentation package in FreeSurfer. Front. Aging Neurosci. 6 261 (2014).

40. Saygin, Z. M. et al. High-resolution magnetic resonance imaging reveals nuclei of the human amygdala: manual segmentation to automatic atlas. Neuroimage 155, 370-382 (2017).

41. Jacobson, $R$. The contributions of sex and drinking history to the $\subset T$ brain scan changes in alcoholics. Psychol. Med. 16, 547-559 (1986).

42. Kroft, C. L. et al. Brain ventricular size in female alcoholics: an MRI study. Alcohol 8, 31-34 (1991).

43. Agartz, I., Shoaf, S., Rawlings, R. R., Momenan, R. \& Hommer, D. W. CSF monoamine metabolites and MRI brain volumes in alcohol dependence. Psychiatry Res. 122, 21-35 (2003).

44. Alfonso-Loeches, S., Pascual, M. \& Guerri, C. Gender differences in alcohol-induced neurotoxicity and brain damage. Toxicology $\mathbf{3 1 1}$ 27-34 (2013)

45. Dale, A. M., Fischl, B. \& Sereno, M. I. Cortical surface-based analysis: I. Segmentation and surface reconstruction. Neuroimage 9, 179-194 (1999).

46. Desikan, R. S. et al. An automated labeling system for subdividing the human cerebral cortex on MRI scans into gyral based regions of interest. Neuroimage 31, 968-980 (2006).

47. Iglesias, J. E. et al. A computational atlas of the hippocampal formation using ex vivo, ultra-high resolution MRI: application to adaptive segmentation of in vivo MRI. Neuroimage 115, 117-137 (2015)

48. Aarts, E., Verhage, M., Veenvliet, J. V., Dolan, C. V. \& van der Sluis, S. A solution to dependency: using multilevel analysis to accommodate nested data. Nat Neurosci. 17, 491-496 (2014).

49. Simes, R. J. An improved Bonferroni procedure for multiple tests of significance. Biometrika 73, 751-754 (1986).

50. Fudge, J. L. \& Tucker, T. Amygdala projections to central amygdaloid nucleus subdivisions and transition zones in the primate. Neuroscience 159, 819-841 (2009).

51. Sciascia, J. M., Reese, R. M., Janak, P. H. \& Chaudhri, N. Alcohol-seeking triggered by discrete pavlovian cues is invigorated by alcohol contexts and mediated by glutamate signaling in the basolateral amygdala. Neuropsychopharmacology 40, 2801-2812 (2015).

52. Ryabinin, A. E., Melia, K. R., Cole, M., Bloom, F. E. \& Wilson, M. C. Alcohol selectively attenuates stress-induced c-fos expression in rat hippocampus. J. Neurosci. 15, 721-730 (1995).

53. Alvarez, I., Gonzalo, L. M. \& Llor, J. Effects of chronic alcoholism on the amygdaloid complex. A study in human and rats. Histol. Histopathol. 4, 183-192 (1989). 
54. Stavro, K., Pelletier, J. \& Potvin, S. Widespread and sustained cognitive deficits in alcoholism: a meta-analysis. Addiction Biol. 18, 203-213 (2013).

55. Pfefferbaum, A., Rosenbloom, M., Deshmukh, A. \& Sullivan, E. V. Sex differences in the effects of alcohol on brain structure. Am. J. Psychiatry 158, 188-197 (2001).
56. Keyes, K. M., Martins, S. S., Blanco, C. \& Hasin, D. S. Telescoping and gender differences in alcohol dependence: new evidence from two national surveys. Am. J. Psychiatry 167, 969-976 (2010).

57. Craparo, G., Ardino, V., Gori, A. \& Caretti, V. The relationships between early trauma, dissociation, and alexithymia in alcohol addiction. Psychiatry Investig. 11, 330-335 (2014). 